\title{
Capillary wetting of profiled polyester fibres - a comparison between macroscopic and microscopic analysis
}

\author{
G Glavan $^{1}$, M Kurečič ${ }^{2,4}$, U Maver $^{3}$, K Stana-Kleinschek ${ }^{2,4}$ and I Drevenšek-Olenik ${ }^{1,5}$ \\ ${ }^{1}$ Faculty of Mathematics and Physics, University of Ljubljana, Jadranska 19, 1000 Ljubljana, Slovenia, \\ ${ }^{2}$ Laboratory for Characterization and Processing of Polymers (LCPP), University of Maribor, Smetanova 17, \\ SI2000 Maribor, Slovenia, \\ ${ }^{3}$ Faculty of Medicine, University of Maribor, Insitute of Biomedical Sciences and Department of Pharmacology, \\ Taborska ulica 8, SI2000, Maribor, Slovenia, \\ ${ }^{4}$ Graz University of Technology, Institute for Chemistry and Technology of Materials, Stremayrgasse 9, AT- \\ 8010, Graz, Austria, \\ ${ }^{5}$ J. Stefan Institute, Jamova 39, SI1000 Ljubljana, Slovenia.
}

E-mail: irena.drevensek@ijs.si

Keywords: Textile fibres, capillary wetting, sorption kinetics, microscale methods

\begin{abstract}
A comparative study between the standard Washburn macroscale method for determination of the capillary wetting rate in a nonwoven textile fibre mesh and the polarization optical video-microscopybased microscale method for investigation of the wetting process in single fibres is reported. The study was performed with profiled polyester (PES) fibres associated with superior wicking performance. Both methods resulted in very similar values of the wetting rate, which confirms that for textile materials based on such type of fibres the microstructure of a single fibre dictates liquid transport properties on the micro- as well as on the macroscale. Consequently, for such materials the microscale method is fully competitive with the standard macroscopic approaches.
\end{abstract}




\section{Introduction}

Some of the most important characteristics of textile materials are the capacity to absorb water-based liquids and the rate of the associated liquid sorption and desorption processes. These properties are customarily determined by standardized instrumental methods relying on macroscopic approaches based on testing patches of a selected fabric [1]. However, transport of water through the fabric is a complex problem that involves capillary effects associated with different structures and length scales, from nano- and micro-pore structure associated with individual fibres through the interstitial channels in the fibrous assembly forming a yarn to the pores present in-between the yarns comprising a woven fabric [2-9]. Due to interconnection of various phenomena it is usually very difficult to resolve how a specific structural parameter of the single fibre affects the kinetics of water transport at different levels [10].

In our recent work we demonstrated that characteristic wetting and sorption times of individual textile fibres can be readily determined by analysis of polarization optical microscopy (POM) images taken during liquid uptake process [11-13]. The analysis is based on temporal modifications (video-miscroscopy) of the intensity of transmitted light passing through the fibre in cross-polarizers configuration. In this work a comparative study between the standard macroscopic method for determination of the wetting kinetics and its video-microscopy-based counterpart is reported. The profiled polyester (PES) fibres used in the study (Coolplus $\left.{ }^{\circledR}\right)$ are associated with textile materials known for their superior wicking performance $[14,15]$. Our results show that both methods give very similar values for the wetting rate, which verifies that for materials based on these fibres the microstructure of a single fibre plays a dominant role in the liquid transport behaviour. Consequently, for such materials the microscale method can be fully competitive with the standard macroscopic approaches.

\section{Experimental procedures}

The nonwoven PES fibre mesh (Cool Plus II, Litia Spinnery, Slovenia) was composed of fibres with a linear density of $1.5 \mathrm{dtex}$ and a staple length of $38 \mathrm{~mm}$. Figure 1a shows the scanning electron 
microscopy (SEM) image of a single fibre. Longitudinal channels associated with the four-lobal (plusshaped) cross-sectional profile are clearly visible on its surface $[16,17]$. The fibre mesh was stored at temperature $T=20^{\circ} \mathrm{C}$ and relative humidity $\eta=65 \%$. Macroscopic physical properties of the material were determined by a standard tensiometer test method used in textile engineering as described elsewhere [18]. Its water retention was found to be $5 \%$ and its contact angle for water was measured to be $86.8^{\circ} \pm 0.8^{\circ}$.

For optical investigations one or more single fibres were pulled out from the mesh and fixed via their ends by a sticky tape to the microscope slide. Then the assembly was covered with a cover slip and both glasses with the fibres in-between were clamped together at their edges by two binder clips (15 mm in length). This produced a pressure of around 1 bar. Figure $1 \mathrm{~b}$ shows a conventional colour image of the sample with three fibres obtained in a configuration in which the transmission directions of the polarizer and the analyser were oriented at $45^{\circ}$ with respect to each other.
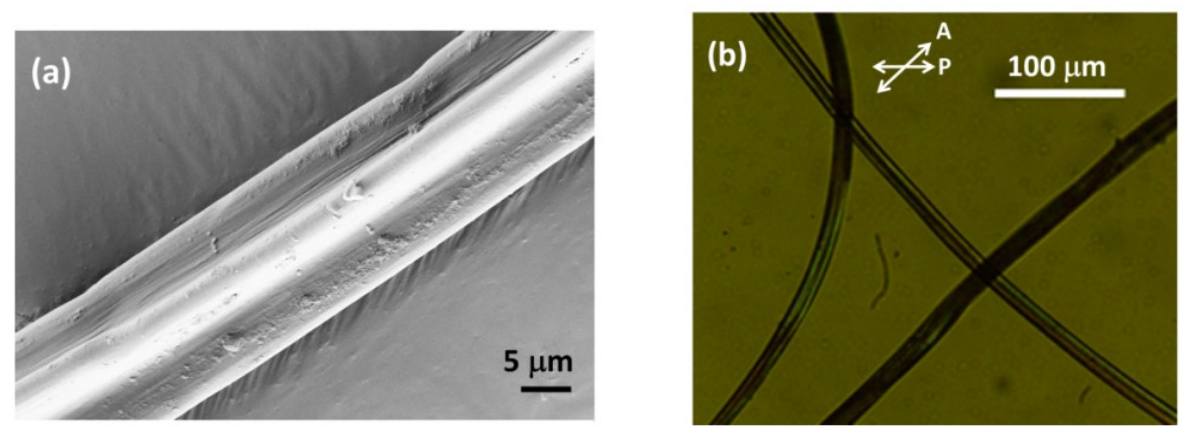

Figure 1. (a) Scanning electron microscopy (SEM) image of a single fibre. Longitudinal surface channels associated with plus-shaped cross-sectional profile can be noticed. (b) Colour polarization optical microscopy image of three fibres placed between two glass plates. The polarizer $(\mathrm{P})$ and the analyser (A) are oriented at $45^{\circ}$ with respect to each other.

To investigate the capillary wetting effect, samples that contained only one fibre were placed on the rotation stage of the microscope (Nikon Optiphot2-pol, Nikon, Japan). The polarizer and the analyser were set into crossed configuration. Then the sample was rotated so that the longitudinal 
direction of the fibre was oriented at $45^{\circ}$ with respect to the transmission axes of the polarizer and the analyser. After that, a drop $(5 \mu \mathrm{l})$ of ultra-pure water (Milli-Q, $18.2 \mathrm{M} \Omega \mathrm{cm}$ at $25{ }^{\circ} \mathrm{C}$ ) produced by the Milli-Q ${ }^{\circledR}$ system (EMD Millipore Corporation, USA) was released at one edge of the gap between both glass plates. The capillary action of the plates rapidly soaked the drop into the gap. Subsequently, wetting of the fibre took place, which caused modifications of the intensity of the light transmitted through the fibre (see figure 2). These modifications were monitored in-situ with a fast monochrome camera (250 fps, IDS UI-3370CP-M-GL, IDS, Germany). Afterwards, analysis of video images was performed in Python (Open Source) to deduce temporal modifications of transmitted intensity $I_{\mathrm{T}}(t)$ and analysis in ImageJ (Open Source) was used to resolve modifications of the fibre diameter $D(t)$. The value of $D$ was obtained as a distance between the two parallel edges of the image of the fibre as resolved by the Canny edge detection algorithm. Its threshold (resolution) was determined by the ratio of the camera pixel size $(5.5 \mu \mathrm{m})$ and the magnification $(20 \mathrm{x})$ and was $0.28 \mu \mathrm{m}$. The value of $I_{\mathrm{T}}$ was obtained by summing up the intensity detected at selected camera pixels and dividing it by the number of the pixels. The resolution of $I_{\mathrm{T}}$ was determined by the dynamic range (gray scale depth) of the camera, which was in our case 8-bit (256 gray-scale values). The analysis was performed for 20 samples.
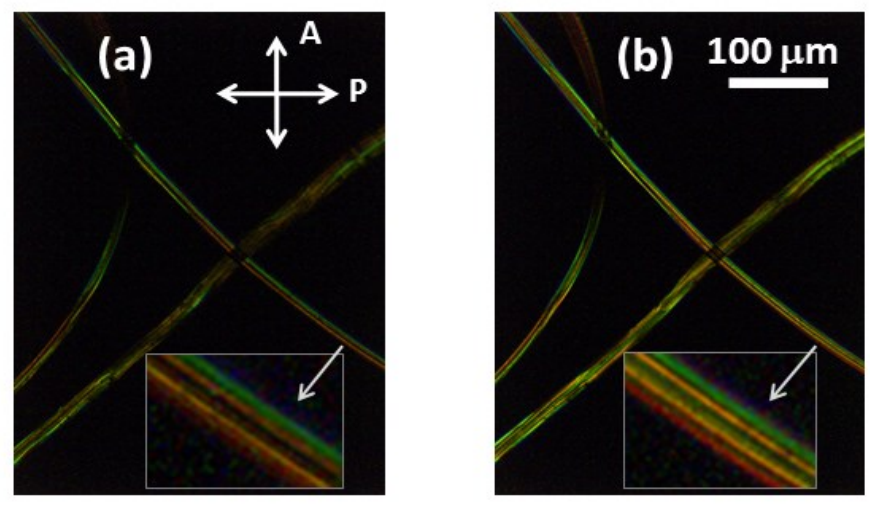

Figure 2. Colour polarization optical microscopy images of the fibres in crossed polarizers configuration: (a) before wetting, (b) after wetting. The insets show magnified images of a single fibre. The intensity of transmitted light increases significantly, while the colours of different longitudinal segments remain more or less the same. 


\section{Background}

Textile filaments and fibres exhibit optical anisotropy (birefringence) because of directional alignment of polymer chains induced by spinning and drawing processes. Due to the symmetry of these processes, the induced anisotropy is uniaxial, i.e. the fibres exhibit only two principal refractive indices: the principal refractive index for light polarized parallel $\left(n_{\mathrm{II}}\right)$ and perpendicular to the fibre axis $\left(n_{\perp}\right)$ [1]. PES fibres are known to be strongly birefringent. The typical value of their optical anisotropy $\Delta n=\left(n_{\mathrm{II}}-n_{\perp}\right)$ is around 0.15 [19]. Consequently they can be readily examined by polarization optical microscopy (POM) using diascopic illumination combined with crossed polarizer and analyser configuration. If the fibre is oriented at $45^{\circ}$ with respect to the transmission axes of the polarizer and the analyser, the intensity of transmitted light $I_{T, b}$ is given as [20]

$$
I_{T, b}=I_{0} \sin ^{2}\left(\frac{\Delta \Phi}{2}\right)
$$

where $I_{0}$ is the intensity of incident light and

$$
\Delta \Phi=2 \pi d \Delta n / \lambda
$$

is the optical retardation, where $d$ is the optical path-length through the fibre and $\lambda$ is the optical wavelength. The average diameter $D$ of PES fibres used in our study is around $10 \mu \mathrm{m}$ (see figure 1a), therefore for visible light $(\lambda \sim 500 \mathrm{~nm})$ the maximal value of $\Delta \Phi$ is around $6 \pi$. Consequently, when light passes through different cross-sectional regions of the fibre associated with different values of $d$ $\leq D$, the value of $I_{T, b} / I_{0}$ oscillates. In the POM images these oscillations show up as a striped coloured pattern parallel to the longitudinal direction of the fibre (see figure 2).

Another property that affects the intensity of light detected by the imaging sensor is the refractive index mismatch between the fibre material and the surrounding medium. This mismatch produces light reflection and refraction at the interface between both media and light scattering from imperfections that are present at the interface [21]. Consequently, a part of the incident light is redirected away from 
the optical axis of the microscope and less light enters the imaging sensor. For that reason, in conventional optical microscopy (without polarizers) or if the polarizer and analyser in the POM are not crossed with each other, the fibres look darker than the surrounding region (see figure $1 \mathrm{~b}$ ). The exact dependence of the transmissivity on the ratio between the refractive indices of the fibre and the refractive index of the surrounding medium $n_{\mathrm{s}}$ depends on the shape and size of structural details with respect to the optical wavelength $\lambda$. In case of small imperfections, scattering can be described by the Rayleigh formula and the transmitted intensity is given as [22]

$$
I_{T, S}=I_{0}\left[1-f\left(\lambda, r_{0}\right) \cdot g(N)\right]
$$

with $g(N)$ defined as

$$
g(N)=\left(\frac{N^{2}-1}{N^{2}+2}\right)^{2}
$$

where $r_{0}$ is the characteristic size of imperfections and $f$ is a function dependent on their size and shape, while $N$ denotes the relative refractive index for specific light polarization $\left(N_{\mathrm{II}}=n_{\mathrm{II}} / n_{\mathrm{s}}, N_{\perp}=\right.$ $\left.n_{\perp} / n_{\mathrm{s}}\right)$. The refractive indices of PES fibres are $n_{\mathrm{II}} \sim 1.70$ and $n_{\perp} \sim 1.54[14,19]$. Therefore, when the fibre is surrounded by air with $n_{\mathrm{s}} \sim 1.0$, the corresponding values of $N_{\mathrm{II}}$ and $N_{\perp}$ are 1.70 and 1.54, respectively. But, when it gets surrounded with a water-based liquid with $n_{\mathrm{s}} \sim 1.33$, these values drop to $N_{\text {II }}=1.28$ and $N_{\perp}=1.16$, respectively. As a result, the value of $g(N)$ averaged over both polarizations drops from 0.12 to 0.02 and light scattering is significantly reduced. Reduction of light scattering is a basic reason why wet textile fabric is usually more transparent than the dry one.

In summary, considering both effects described above, when some liquid is put in contact with a textile fibre the brightness of a selected segment of the fibre in the POM image is expected to either increase or decrease due to modification of $\Delta \Phi$ and to increase due to reduction of optical reflection and scattering associated with decrease of $g(N)$.

\section{Results and discussion}

The analysis of the transmitted optical intensity was performed in four different neighbouring 
regions of the sample image. Figure 3 shows temporal variations of the average intensity of these regions calculated as

$$
<I_{T}(t)>=\frac{1}{S} \int I_{T}(x, y, t) d x d y
$$

where the integration took place over the selected region (area j) and $S$ is the surface area of that region. Water flow entered the imaged area from the upper right side. The value $t=0$ is set so that it coincides with the star of the changes taking place in area 1 of the image. The initial drop of $\left\langle I_{\mathrm{T}}\right\rangle$ is attributed to capillary flow between the hydrophilic glass plates that causes a rapid pressure increase around the fibre and subsequently leads to lateral shrinking of the fibre. Due to the shrunk relative area of the light transmitting fibrous material, less light comes to the image detector and so $\left\langle I_{\mathrm{T}}>\right.$ decreases.

The initial dip is followed by an increase of $\left\langle I_{\mathrm{T}}>\right.$, which is attributed to capillary flow between the fibre and the glass plates. As discussed in the previous section, for a specific segment of the fibre (with specific value of $d$ ) this can lead either to an increase or a decrease of the transmitted intensity (Eq. 1). However, because in our experiments light transmitted through a large area of the fibre associated with different values of $d$ was measured, the oscillations due to modifications of the optical retardation $\Delta \Phi$ averaged out. Consequently, the observed increase of $\left\langle I_{\mathrm{T}}\right\rangle$ is predominantly attributed to the decrease of light scattering.

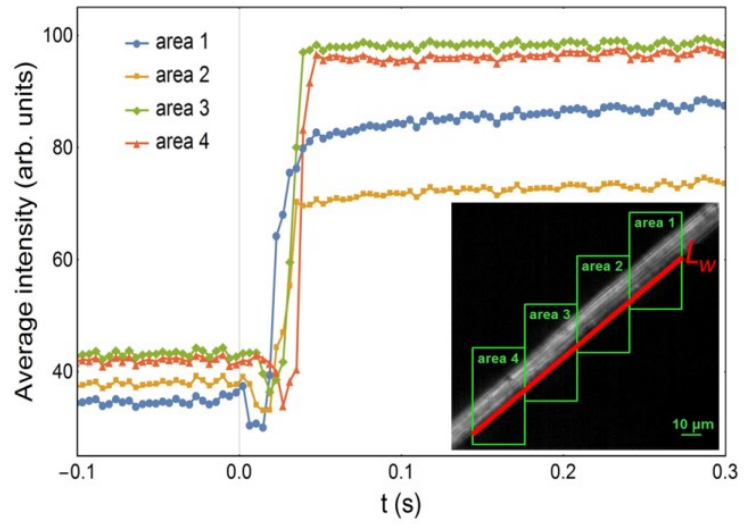


Figure 3. Temporal modifications of average intensity of the transmitted light detected in four different image areas. The drop of water was injected from the upper right part of the image. The inset shows the corresponding monochrome image of the fibre and allocation of the four analysed areas. Red line indicates the total fibre length spanning from the area 1 to the area 4 , which is $L_{w}=140 \mu \mathrm{m}$.

The capillary wetting process is accompanied with modifications of the apparent diameter (thickness) $D$ of the fibre. The corresponding dependence of $\langle D(t)\rangle$ averaged over all four investigated areas is shown in figure 4. The initial shrinkage is followed by a subsequent expansion. Expansion occurs when longitudinal channels on the surface of the fibre (see figure 1a) are filled up with water. The major part of the modifications takes place within $\tau_{\mathrm{w}} \sim 0.05 \mathrm{~s}$, which defines the characteristic capillary wetting time of the $L_{\mathrm{w}}=140 \mu \mathrm{m}$ long segment of the fibre extending from the upper corner of the area 1 to the lower corner of the area 4 (see inset of figure 3). The average microscale capillary wetting rate is hence $<v_{\text {mic }}>=\left(L_{\mathrm{w}} / \tau_{\mathrm{w}}\right) \sim 3 \mathrm{~mm} / \mathrm{s}$. Very similar values were obtained also in other samples exhibiting the above described behaviour.

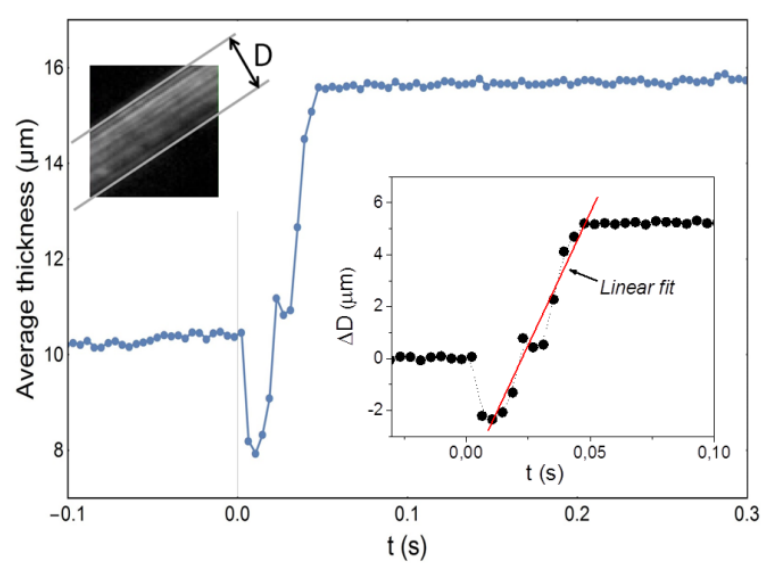

Figure 4. Temporal modifications of apparent thickness $D$ of the fibre exposed to water at $t=0$. The upper left insert shows POM image of the fibre and the attributed parameter. The lower right insert 
shows a magnified region of the graph associated with the capillary wetting event. The corresponding wetting time is $0.05 \mathrm{~s}$.

The behaviour shown in figures 3 and 4 was observed only in about $15 \%$ of the investigated cases. The most frequently observed performance was the one shown in figure 5 . In this case both, the average intensity $\left\langle I_{\mathrm{T}}>\right.$ (figure 5a) and the average thickness $\langle D>$ (figure $5 \mathrm{~b}$ ), alter with time in a multi-step manner. The plateau regions between the steps are attributed to the time needed for water to penetrate from the outer area of the plus-shaped fibre to the inner channels of the fibre, as illustrated by the drawings in the inset of figure $5 \mathrm{~b}$. The total duration time of modifications associated with all steps is around $0.05 \mathrm{~s}$, similar to the single-step behaviour discussed previously.
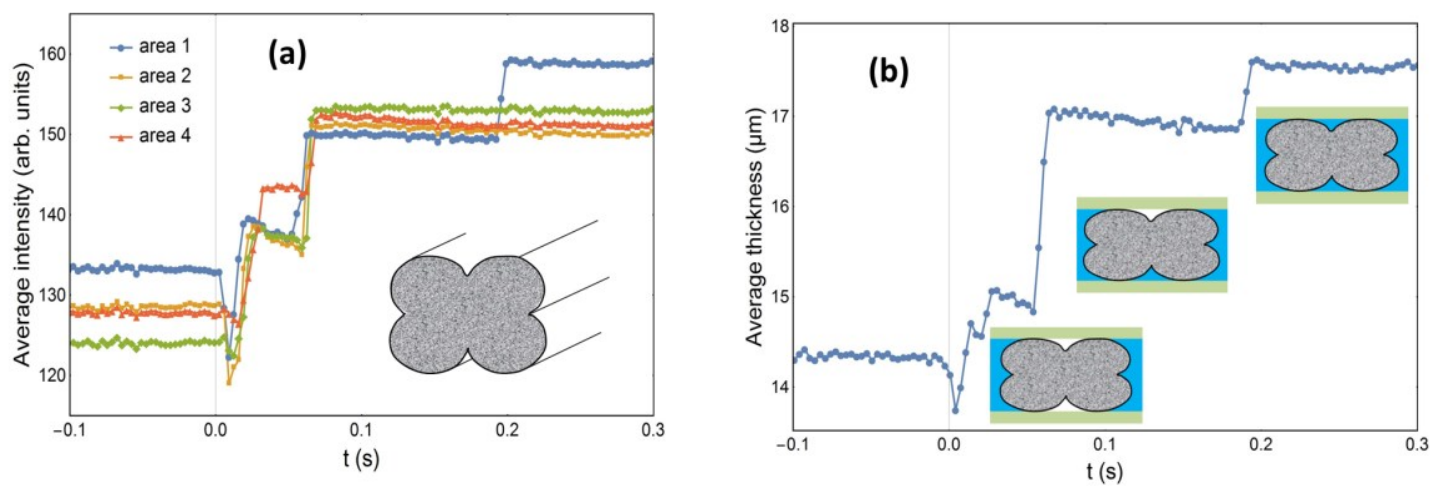

Figure 5. (a) Modifications of average intensity of transmitted light through a single PES fibre observed during the capillary wetting process. The inset illustrates plus-shaped cross sectional profile of the fibre. (b) Modifications of the average thickness of the fibre. Schematic drawings illustrate wetting stages associated with the observed multi-step behaviour. Green regions indicate glass, blue water and white air, respectively.

The macroscopic process of water sorption into nonwoven fibre mesh was found to be rather 
slow. Figure 6 shows the time dependence of the squared mass $m^{2}$ of absorbed water measured by the standard Washburn method employing a glass tube filled with the compressed fibre mesh (syphon test) [23]. The dry mesh had a density of $0.45 \mathrm{~g} / \mathrm{cm}^{3}$, which means that after water sorption about $1 / 3$ of the inner volume of the tube was occupied by PES fibres, while about $2 / 3$ of the volume was filled with water. Consequently, the corresponding time dependence of the height of the water front $h(t)$ (see inset of figure 6) can be calculated as:

$$
h(t)=\frac{m(t)}{\varepsilon_{w} \rho S_{0}} \quad,
$$

where $\varepsilon_{w}=2 / 3$ is the volume fraction of water, $\rho=1.0 \mathrm{~g} / \mathrm{cm}^{3}$ is the density of water and $S_{0}$ is the cross-sectional area of the tube that was in our case $0.78 \mathrm{~cm}^{2}$. The characteristic Washburn-type linear dependence of the square mass on time, denoted by the red line in figure 6 , is observed in the interval $0<t<40 \mathrm{~s}$. In this time interval the water front rises from $h(t=0)=0$ to $h(t=40 \mathrm{~s})=4.0 \mathrm{~cm}$, which results in an average macroscale capillary wetting rate $<v_{\mathrm{mac}}>=(\Delta h / \Delta t)=1 \mathrm{~mm} / \mathrm{s}$.

At this point one needs to stress out that due to nonlinear behaviour of $h(t)$, i.e. $h(t) \propto t^{1 / 2}$, the average capillary wetting rate depends on the measurement time $\Delta t$ and in general increases with decreasing value of $\Delta t$. Therefore to obtain the value that can be considered characteristic for a specific system, one should choose a time interval that is in some way intrinsic to the system. In our case this interval was selected to be the interval of the initial linear increase of $m^{2}$ with $t$.

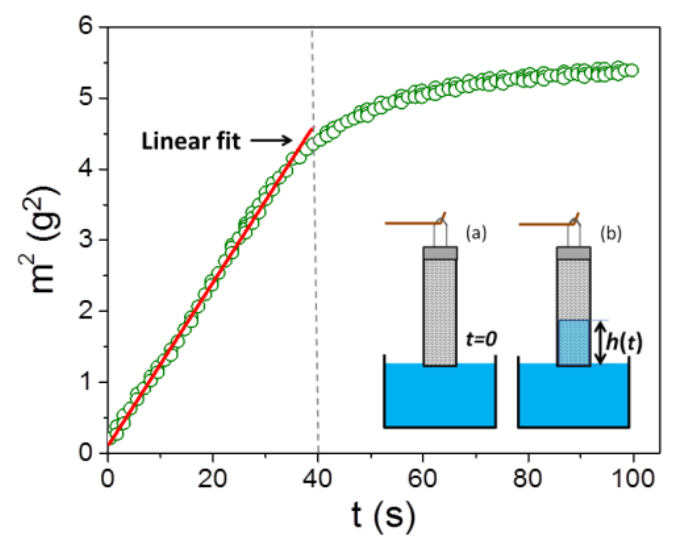


Figure 6. Kinetics of water sorption measured by the standard Washburn method (syphon test) employing a glass tube filled with the compressed fibre mesh. Squared mass of adsorbed water is given as a function of sorption time. The inset shows a schematic drawing of the method.

The obtained values of the macroscale and microscale capillary wetting rates are very similar $\left(<v_{\text {mac }}>=1 \mathrm{~mm} / \mathrm{s},<v_{\text {mic }}>=3 \mathrm{~mm} / \mathrm{s}\right)$. The agreement is actually even better than can be deduced from the results. Namely, one needs to take into account that in the microscale experiments the capillary flow occurs in channels surrounded by heterogeneous walls, i.e. the capillary force partially originates from the water-glass interface and partially from the water-PES interface (see figure 5b). Because the contact angle of water on glass is close to zero, capillary wetting rate in such conditions is larger than in channels surrounded only by PES, as is the case in the macroscale measurements.

\section{Conclusions}

The observed good matching between the kinetices of macroscale and microscale capillary wetting phenomena in the investigated material is attributed to low swelling level of the fibres $(5 \%)$, due to which the capillary-bound water strongly dominates the swelling-bound water [24]. Consequently, the swelling process, which usually results in a relatively slow microscale sorption [13], is negligible and capillary wetting is predominantly driven by the capillary flow alongside the surface channels associated with the plus-shaped cross-sectional profile of the fibre. This gives the corresponding fabrics their superior wicking behaviour.

Despite the fact that in our study both methods gave very similar final results, they in general probe very different levels of the capillary wetting process. There are also large methodological differences between them. The main advantage of the macroscale Washburn method is that microscale diversity associated with polydispersity of fibre sizes and shapes mainly averages out. So only few measurements are needed to obtain a suitable certainty of the measured properties. The main 
disadvantage is poor control over structural arrangement and homogeneity of the fibre mesh in the measurement tube. The microscale POM-imaging-based method, on the other hand, requires analysis of a large number of samples, so that a suitable statistical average with respect to the fibre diversity is obtained. To make good testing samples with improved reproducibility, one should use a clamping system with controllable pressure. Also the release of water droplet onto the sample should be automated. Besides this, the time required for measurements can be considerably reduced if not only one, but several fibres are introduced into one and the same testing cell at large enough distances between them. Further optimization requires development of reliable image analysis procedures that have to be adjusted for the specific type of the fibres investigated $[13,25]$. The main advantage of the microscale method is that some external parameters, such as pressure, temperature, chemical reactants or some geometrical barriers, can be varied and controlled relatively easily, so it provides a very convenient platform for the investigation of the effect of those parameters on the wetting characteristics of single fibres.

\section{Acknowledgements}

The authors acknowledge financial support of the Slovenian research program P1-092 ("Light and Matter") and P2-0118 (“Textile Chemistry"), as well as national research project L2-5492. The authors would also like to acknowledge Litia Spinnery for kindly supplying fibrous materials.

\section{References}

[1] Hearle J W S and Morton W E 2008 Physical Properties of Textile Fibres 4th edn (Woodhead Publishing)

[2] Birrfelder P, Dorrestijn M, Roth C and Rossi R M 2013, Effect of fiber count and knit structure on intra- and inter-yarn transport of liquid water Text. Res. J. 83 1477-1488

[3] Chatterjee A and Singh P 2014 Studies of wicking behaviour of polyester fabric J. of Textiles 379731 
[4] Hamdaoui M, Achour N S and Nasrallah S B 2014 The influence of woven fabric structure on kinetics of water sorption J. Eng. Fiber. Fabr. 9 101-106

[5] Kissa E 1996 Wetting and wicking Text. Res. J. 66, 660-668

[6] Perwuelz A, Mondon P and Caze C 2000 Experimental study of capillary flow in yarns Text. Res. J. 70, 333-339

[7] Kumar B and Das A 2014 Vertical wicking behaviour of knitted fabrics Fibers and Polymers 15, 625-631.

[8] Zhu L, Perwuelz A, Lawandowski M and Campagne C 2008 Static and dynamic aspects of liquid capillary flow in thermally bonded polyester nonwoven fabrics J. Adhes. Sci. Technol. 22, 745-760 [9] Kim S H, Lee J H, Lim D Y and Jeon H Y 2003 Dependence od sorption properties of fibrous assemblies on their fabrication and material characteristics Text. Res. J. 73, 455-460.

[10] Hasan M M B, Calvimontes A, Synytska A and Dutschk V 2008 Effect of topographic structure on wettability of differently woven fabrics Text. Res. J. 78, 996-1003

[11] Devetak M, Skoporc N, Rigler M, Peršin Z, Drevenšek-Olenik I, Čopič M and Stana-Kleinschek K 2012 Effect of plasma treatment on water sorption in viscose fibres Mater. Tehnol. 46, 69-73

[12] Devetak M, Peršin Z, Stana-Kleinschek K and Maver U 2014 Utilization of optical polarization microscopy in the study of sorption characteristics of wound dressing host materials Microsc.

Microanal. 20, 561-565

[13] Peršin Z, Devetak M, Drevenšek-Olenik I, Vesel A, Mozetič M and Stana-Kleinschek K 2013 The study of plasma's modification effects in viscose used as an absorbent for wound-relevant fluids Carbohydr. Polym. 97, 143-151

[14] Palenik S J 2003 Microscopical Examination of Fibers in Robertson J and Grieve M eds Forensic Examination of Fibers, 2nd edn (Taylor \& Francis, London)

[15] Bagherzadeh R, Gorji M, Latifi M, Payvandy P and Kong L X 2012 Evolution of moisture management behaviour of high-wicking 3D warp knitted spacer fabrics Fibers Polym. 13, 529-534 [16] Feng A and Zhang Y, 2015 Study of moisture absorption and sweat discharge of honeycomb polyester fiber IOP Conf. Series: Mat. Sci. Eng. 87, 012095 


\section{Capillary wetting of profiled polyester fibres}

[17] Gorji M and Bagherzadeh R 2016 Moisture management behaviours of high wicking fabrics composed of profiled fibres Indian J. Fibre Text. Res. 41, 318-324

[18] Peršin Z, Stana-Kleinschek K, Sfiligoj Smole M and Kreže T 2004 Determining the surface free energy of cellulose materials with the powder contact angle method Text. Res. J. 74(1), 55-62

[19] Gorski A, McCrone W C 1998 Birefringence of fibers Microscope 46, 3-16

[20] Rajh D, Shelestiuk S, Mertelj A, Mrzel A, Umek P, Irusta S , Zak A and Drevenšek-Olenik I 2013 Effect of inorganic 1D nanoparticles on electrooptic properties of 5CB liquid crystal Phys. Status Solidi A 210, 2328-2334

[21] Peng B, Ding T H and Wang P 2012 Propagation of polarized light through textile materials Appl. Opt. 51, 6325-6334

[22] Born M and Wolf E 2003 Principles of Optics 7th edn (Cambridge University Press).

[23] Washburn E W 1921 The dynamics of capillary flow Phys. Rev. 17, 273-283.

[24] Smirnova E E, Perepelkin K E, Smirnova N A and Brezgina S A 2002 Change in the properties of textile materials containing polyester and cellulose fibres caused by moisture Fibre Chemistry 34, 192-196

[25] Parada M, Derome D, Rossi R M and Carmeliet J 2017 A review on advanced imaging technologies for the quantification of wicking in textiles Text. Res. J. 87, 110-132. 